\title{
RADIOMETRIC RECEIVER FOR PASSIVE MICROWAVE IMAGING SYSTEM
}

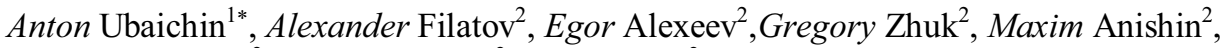 \\ Stanislav Gazitov ${ }^{2}$, Sergey Tarasov ${ }^{2}$, Boris Utkin $^{2}$ \\ ${ }^{1}$ Tomsk Polytechnic University, Institute of Non-Destructive Testing, 634050, Tomsk, Russia \\ ${ }^{2}$ Tomsk State University of Control Systems and Radioelectronics, 634050, Tomsk, Russia
}

\begin{abstract}
The paper describes a new method to develop a zero-type $W$ band radiometric receiver. The block diagram and operating algorithm of the digital control system are presented. The main advantages of the zero method in passive radio physical investigations in comparison with classical engineering solutions are shown.
\end{abstract}

\section{Introduction}

Passive thermal radio imaging systems are currently being in demand. An object can be detected by radio thermal features without active radio emission. The passive thermal radar system operates regardless of the weather and time of day as compared to the equipment based on infrared methods. Therefore, these systems can be used in navigation in conditions of restricted visibility (aviation security), for radiolocation of unmanned aviation vehicles, in environmental monitoring, for medical applications and etc. Over the last years, much attention has been paid to the radio systems used for imaging of objects hidden behind not optically transparent media due to increased terrorism threat [1].

\section{Study objectives}

Over the last 40 years, the radio imaging system has become sufficiently advanced due to improved microwave devices: antennas with low loss and high directivity, special processing algorithms in real time and etc. However, no significant techniques were found to improve the design of radiometric receivers. The radiometric receivers currently used are based on total power or modulation principle proposed by Dick's in 1946 [2]. It is wellknown, that the receiver gain fluctuation has a major impact on sensitivity of these receivers. The gain fluctuation has a maximum effect near the zero frequency and its spectral density decreases by $1 / \mathrm{f}$ law (similar to flicker noise). Increase in the averaging time in this type of radiometric receivers is a good approach to improve the fluctuation sensitivity. As a result, the impact of low frequency gain fluctuations within the correlation time at different frequencies becomes less significant [3].Therefore, in most radiometric systems, the decreased measurement time inevitably leads to sensitivity degradation.

*Corresponding author:ubaychin@tpu.ru 


\section{Theory}

The use of zero-type radiometric receiver decreases the averaging time and maintains the sensitivity at nominal level due to insignificant effect (theoretically null) of gain fluctuations on measurement results. However, the use of the zero measurement method in the radio imaging system is constrained due to the following reasons. The zero-type radiometer is a particular type of the modulation radiometer. It employs symmetry amplitude pulse modulations of both the fiducial noise source and antenna noise similar to that in the modulation radiometer. The difference is that the fiducial noise source is adjustable and it is tuned to the antenna signal through the feedback loop. In this case, the linearity of the radiometric system depends on the linearity of the fiducial noise source feedback loop, rather than that of the receiver. This makes the system more complicated and causes feedback loop fault and system delay [4]. In addition, it increases the requirements to the fiducial source linearity. The modified zero measurement method used in the radiometric receiver, which allows increase in the measurement rate without fluctuation sensitivity loss, is described in [5]. The modification implies the use of two types of synchronous processing pulse modulations, width and amplitude ones.

\section{Experimental researches}

Figure 1 shows the block diagram of the radiometric receiver with 3-mm wavelength used in the passive imaging system based on the modified zero-type method. It decreases the measurement time with given both sensitivity and long time stability, and eventually, it improves the image frequency rate.

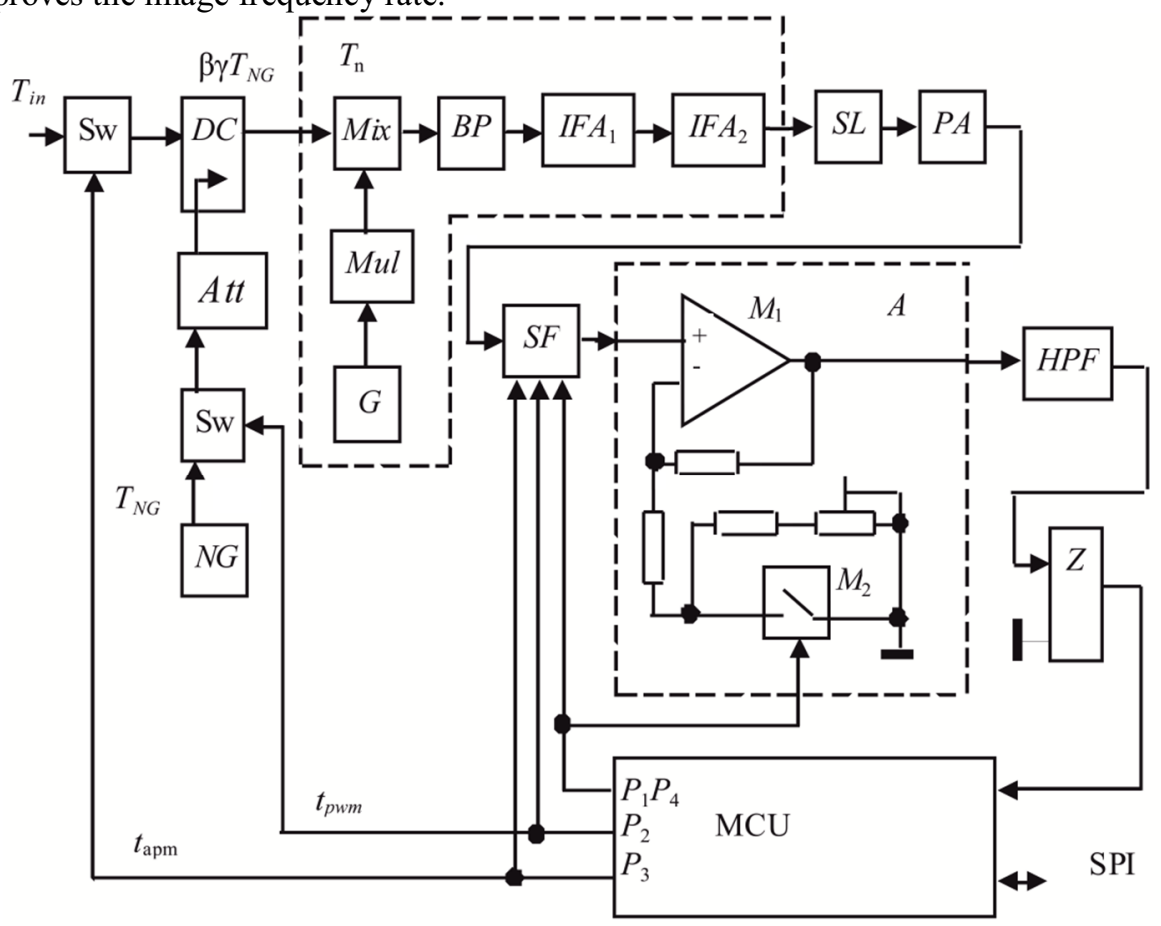

Figure 1. Block diagram of the radiometric receiver.

A directional coupler DC is placed at the receiver input and the pulse-width signal of noise generator NG modulated by microwave switch $\mathrm{Sw}_{2}$ is the input of the directional 
coupler. The level of the signal is adjusted by attenuator Att during the radiometric system calibration. The amplitude modulation is processed by microwave switch $\mathrm{Sw}_{1}$. The receiver designed in the heterodyne circuit consists of generator $\mathrm{G}$ with frequency of $45.5 \mathrm{GHz}$, frequency multiplier (doubler) $\mathrm{M}$ and mixer Mix. Thus, the $91 \mathrm{GHz}$ signal is fed to the mixer. The output signal of the mixer passes through the band pass filter with band pass $2 \ldots 4 \mathrm{GHz}$ to the input of two serially connected intermediate frequency amplifiers IFA $\mathrm{A}_{1}$ and IFA $_{2}$ with the total gain of about $61 \mathrm{~dB}$. Thus, the operating bandwidth of the receiver is 93...95 GHz. A square law detector SLD is placed at the output of the second intermediate frequency amplifier. The output signal of the detector is equal to the square of the input voltage, hence, to the input power. The square law detector based on the Schottky diode is designed according to the "diode is the current to voltage converter" circuit. Its sensitivity is about $10 \mathrm{uW} / \mathrm{V}$. The post-detector section consists of preamplifier PA, synchronous low pass filter SF and invert amplifier A with discrete adjustable gain. The next components are conventional modules for zero-type receiver realization: high pass filter HPF, which excludes the direct current, and analog zero-crossing comparator $\mathrm{Z}$.

The operating principle of the radiometric receiver is as follows. A pulse signal for amplitude modulation from the microcontroller output to the control $\mathrm{Sw}_{1}$ input is a periodic serial pulse with duration $t_{\mathrm{apm}}$ and duty cycle 2 . The signal of the noise generator in the noise adding channel is pulse-width modulated by $\mathrm{Sw}_{2}$ that is controlled by $t_{\mathrm{pwm}}$. Thus, there are three noise signal levels at the receiver input:

-while pulses $t_{\mathrm{apm}}$ and $t_{\mathrm{pwm}}$, signal A equal to $\beta \gamma T_{\mathrm{NG}}+T_{N}$;

-while only $t_{\text {apm }}$ pulse, signal B equal to $T_{\mathrm{N}}$;

-without both $t_{\text {apm }}$ and $t_{\mathrm{pwm}}$, signal C equal to $T_{\mathrm{in}}+T_{\mathrm{n}}$

where $T_{\text {in }}$ is the noise temperature at the system input, $T_{N G}$ is the noise temperature of the generator fiducial noise, $T_{n}$ is the amplifier noise, $\beta$ is the coupling factor of the directional coupler, $\gamma$ is loss in the attenuator. Signal $T_{\mathrm{n}}{ }^{\prime}$ is the subject of careful consideration. This signal is the input of the mixer while $\mathrm{Sw}_{1}$ is off, and it is the sum of three signals: $T_{\mathrm{n}}{ }^{\prime}=T_{\mathrm{n}}+T_{\mathrm{n}} R+T_{\text {ninv }}$. The first term $T_{\mathrm{n}}$ is the noise temperature of the receiver. The second term is the same signal, but reflected with $R$ coefficient by $\mathrm{Sw}_{1}$ while it is off. The third term $T_{\text {ninv }}$ is the result of interference of the receiver noises and these noises reflected from Sw1. One way to reduce this interference is fulfillment of the condition $s \geq C_{0} / 2 \sqrt{ } \varepsilon d f$, where $s$ is the electrical length of the waveguide between the $\mathrm{Sw}_{1}$ output and the mixer input, $C_{0}$ is the speed of light in vacuum, $d f$ is the receiver bandwidth and $\varepsilon$ is the electric permittivity. The gain of the amplifier $\mathrm{A}$ is adjusted during calibration by variation of the dividing coefficient when $\mathrm{M}_{2}$ is off (see Figure 1).

$$
\alpha T_{\mathrm{n}}{ }^{\prime}=T_{\mathrm{n}} .
$$

As noted above, the operating principle of the radiometric receiver is based on the modified zero measurement method. According to this modification, the antenna noise temperature is determined by the duration of the pulse-width modulated signal, when zero balance is established, by the following expression [5]:

$$
t_{\mathrm{pwm}}=(C-B) t_{\mathrm{apm}} /(A-B),
$$

where $A, B$ and $C$ are the mean of the signal levels considered above. After substitution of $A, B$ and $C$ in (2) and considering (1), we obtain

$$
t_{\mathrm{pwm}}=T_{\mathrm{in}} t_{\mathrm{apm}} / \beta \gamma T_{\mathrm{NG}},
$$

The last formula (3) implies that the antenna signal is determined indirectly by the duration of the pulse-width modulation, and for this, only one fiducial noise source is used. 
The new 3-mm range radiometric receiver was designed for theory, block diagram and operating algorithm confirmation. The calibration is a one of the experimental research stages of the designed radiometric receiver. The calibration is carried out by classic technique, and consists in alternately connection of afiducial noise sources to the receiver input. Due to the complexity of the fiducial noise source for millimeter wave range, external sources, for example, clear sky, special prepared absorptive materials and etc are used for two point calibration. To implementation of the calibration were used two external noise sources - "cold" fiducial is the sky in clear weather, "hot" fiducial is special prepared absorptive Carbone-based material, which was pre-placed in the volume with temperature control for a long period of time, sufficient to uniform heating of absorptive material.

The series of experimental research is carried out after the calibration. To execute this, the radiometric receiver is placed into the volume with temperature control, able to set negative and positive temperature, operating in the cyclic mode between two target values of the temperatures. The input of the receiver with antenna is aimed to the "hot" fiducial, which placed outside the temperature control volume, thus, in the receiver input is the signal with noise power close to $300 \mathrm{~K}$. The internal temperature of the temperature control volume is changing with specified speed to determine the temperature stability of measurement results. During the cyclic temperature changing the receiver operated in three modes - full power, modulation and zero. At the changes of the temperature from $+25^{\circ} \mathrm{C}$ to $-5^{\circ} \mathrm{C}$, the error corresponds to full power mode is $12.7 \%$, modulation mode is $6.1 \%$ and zero mode is $1.9 \%$.

Figure 2 shows the dependence of output radiometer signals operation in three modes from the time, when the temperature is cyclic changed from $+25^{\circ} \mathrm{C}$ to $+10^{\circ} \mathrm{C}$.

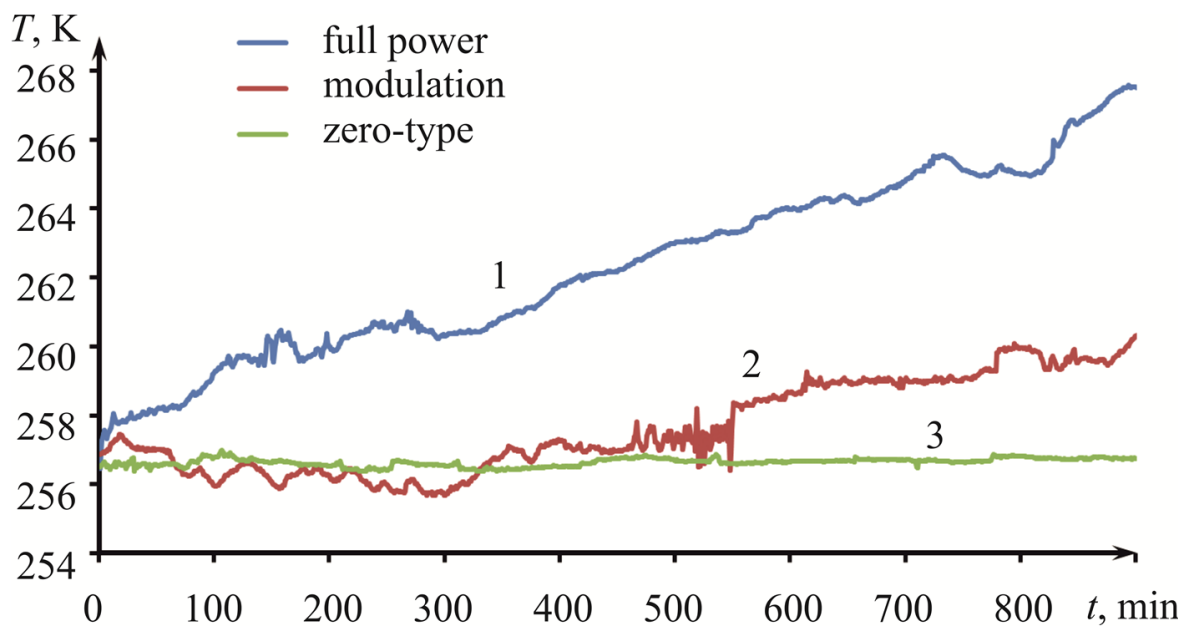

Figure 2. The influence of cyclic temperature changing to the output signals of radiometer in full power (1), modulation (2) and zero (3) modes.

\section{Discussion}

As should be expected, a change of operating temperature is main influence to the measurement accuracy. As shown in conducted experiments, the radiometric receiver has a best long time and temperature stability in the zero operating mode, it is fully corresponds to researches conducted earlier [5]. General analysis of Figure2 shows the some down shifting of the noise temperature scale. It is caused by error in the determination of the noise temperature of the "cold" fiducial or loss in medium between antenna and "hot" 
fiducial during the experimental research. These factors have no significant influence to the measurement results due to normalization of the dynamic range.

\section{Conclusion}

The proposed technical solution significantly simplifies the receiver input high-frequency section. It becomes similar to that of the classical modulation radiometer based on the differential measurement method. The modulation radiometer is used to develop systems for various applications due to simple technical realization of the input high-frequency section and decreased receiver noise temperature effect within the measurement interval. In spite of this, the effect of the drift and fluctuation of the receiver gain on measurement results is great, because the gain is a slope of the radiometer transfer characteristics [5]. In the described zero-type microwave radiometer based on the zero measurement method, the effect of gain fluctuation is minimized, and the slope is determined by a high precision resistive divider. Thus, the circuits are simplified and measurement accuracy is achieved.

\section{Acknowledgments}

The reported study was partially supported by RFBR, research project No. 15-07-04971A.

\section{References}

[1] A. Camps, J. Tarongi, Rem. Sens. 2, 1826 (2010)doi:10.3390/rs2071826

[2] M. Kaisti, M. Altti, T. Poutanen, Remote Sens. 8, 85 (2016), doi:10.3390/rs8020085

[3] X. Lu, J. Li, F. Tao, P. Duan, MATEC Web of Conferences 44, 02084 (2016) doi:10.1051/matecconf/20164402084

[4] A. Bespalko, A. Surzhikov, L.Yavorovich, P. Fedotov, Russ. J. Nondestr. Test. 48, 221 (2012) doi: 10.1134/S1061830912040043

[5] A. Filatov, A. Ubaichin,Meas. Tech. 54, 1160 doi:10.1007/s11018-012-9865-6 